Pacific Journal of Mathematics

ON EQUIVARIANT FUNCTION SPACES 


\title{
ON EQUIVARIANT FUNCTION SPACES
}

\author{
Jesper Michael Møller
}

\begin{abstract}
Some basic features of the homotopy theory of mapping spaces are generalized to an equivariant setting.
\end{abstract}

1. Introduction. The aim of this paper is to extend some well-known theorems about mapping spaces to spaces of equivariant maps. Along the way we consider Bredon cohomology with local coefficients and Postnikov resolutions of equivariant fibrations.

For a finite group $G$, we begin by defining Bredon cohomology $H_{G}^{*}$ with local coefficients. Obstructions to equivariant sections of $G$-fibrations lie in these cohomology groups and the associated classifying $G$-fibrations are thus steps on equivariant Postnikov ladders. See Section 4 for these $G$-Postnikov resolutions and see the preceding sections for the definition of $H_{G}^{*}$ and the construction of the associated classifying $G$-fibrations.

In Section 5 we consider spaces of equivariant sections of $G$-fibrations. By resolving the target fibration, we obtain an equivariant, relative, and twisted version of the Federer spectral sequence converging to the homotopy of the space of equivariant sections. As in the nonequivariant case, this spectral sequence implies nilpotency of spaces of $G$-sections in certain cases. Fibrewise, equivariant localization of the target induces localization of the section space.

Throughout this paper, $G$ denotes a finite (discrete) group with orbit category $\mathscr{O}_{G}$ [1]. I write $K \leq G$ to indicate that $K$ is a subgroup of $G$.

2. Local coefficients in $G-C W$ complexes. In this section equivariant Bredon cohomology is introduced as a framework for equivariant obstruction theory.

Denote by $\mathscr{L}$ the category whose objects are pairs $(X, L)$ with $X$ a (compactly generated) space and $L$ a local coefficient system on $X$. A morphism $\varphi:\left(X_{1} ; L_{1}\right) \rightarrow\left(X_{2}, L_{2}\right)$ in $\mathscr{L}$ is a pair $\varphi=\left(\varphi_{1}, \varphi_{2}\right)$ consisting of a continuous map $\varphi_{1}: X_{1} \rightarrow X_{2}$ and a morphism $\varphi_{2}: L_{1} \rightarrow \varphi_{1}^{*} L_{2}$ of local coefficient systems on $X_{1}$; see [20]. 
Furthermore, for any $G$-space $X$, let $\Phi(X): \mathscr{O}_{G} \rightarrow$ Top be the fixed point set system ([2], p. 275) of $X$, and let $F: \mathscr{L} \rightarrow$ Top denote the forgetful functor.

Definition 2.1. A local $G$-coefficient system on a $G$-space $X$ is a contravariant functor

$$
\underline{\mathscr{M}}: \mathscr{O}_{G} \rightarrow \mathscr{L}
$$

such that $F \underline{\mathscr{M}}=\Phi(X)$.

We shall often use the notation

$$
\begin{aligned}
\underline{\mathscr{M}}(G / H) & =\left(X^{H}, \underline{\mathscr{M}}(G / H)\right), \\
\underline{\mathscr{M}}(\hat{g}) & =(g, M(\hat{g}))
\end{aligned}
$$

where $\underline{M}(G / H)$ is understood to be a local coefficient system on $X^{H}, \hat{g}: G / H \rightarrow G / K$ is left multiplication by $g, g^{-1} H g \subset K$, and $M(\hat{g}): \underline{M}(G / K) \rightarrow g^{*} \underline{M}(G / H)$ a morphism of local coefficient systems on $X^{K}$.

ExAmple 2.2. Let $P: Y \rightarrow B$ be a $G$-fibration in the sense of Bredon [1]. Then $p^{K}: Y^{K} \rightarrow B^{K}$ is an ordinary Serre fibration for each subgroup $K \leq G$ and hence the $i$ th homotopy groups of the fibres, if connected and simple spaces, define an ordinary local coefficient system $\pi_{i}\left(\mathscr{F}^{K}\right)$ on $B^{K}$. Moreover, if $g \in G$ and $g^{-1} H g \subset K$, then left translation by $g$ is a fibre map $g: p^{K} \rightarrow p^{H}$ and the induced maps

$$
g_{*}: \pi_{i}\left(\left(p^{K}\right)^{-1}(b)\right) \rightarrow \pi_{i}\left(\left(p^{H}\right)^{-1}(g b)\right), \quad b \in B^{K},
$$

constitute a morphism

$$
\pi_{i}\left(\mathscr{F}^{K}\right) \rightarrow g^{*} \pi_{i}\left(\mathscr{F}^{H}\right)
$$

Hence the functor

$$
\pi_{i}(\mathscr{F}): \mathscr{O}_{G} \rightarrow \mathscr{L}
$$

given by $\pi_{i}(\mathscr{F})(G / K)=\left(B^{K}, \pi_{i}\left(\mathscr{F}{ }^{K}\right)\right)$ and $\underline{\pi}_{i}(\mathscr{F})(\hat{g})=\left(g, g_{*}\right)$ is a local $G$-coefficient system on $B$.

Let $X$ be a $G-C W$-complex and $\mathscr{M}$ a local $G$-coefficient system on $X$. Since $G$ is finite, each fixed point set $X^{K}, K \leq G$, is an ordinary sub- $C W$-complex [10], and as such it carries for each $n \geq 0$ a cellular cochain group $\Gamma^{n}\left(X^{K} ; \underline{M}(G / K)\right)$ with local coefficients $\underline{M}(G / K): \Gamma^{n}\left(X^{K} ; \underline{M}(G / K)\right)$ is the group ([20], p. 287) of all functions $c$ which to each $n$-cell $E_{\alpha}^{n}=\left(E^{n} \stackrel{h_{q}}{\rightarrow} X_{n}^{K}\right)$ assigns an element $c\left(E_{\alpha}^{n}\right)=\underline{M}(G / K)\left(z_{\alpha}\right)$ where $z_{\alpha}=h_{\alpha}\left(e_{0}\right)$ is the image of the base point $e_{0} \in E^{n}$. 
In order to obtain a useable cohomology group we must demand that these functions for all fixed point spaces behave well under left translations by elements of $G$. This motivates

Definition 2.3. $\Gamma_{G}(X ; \underline{M})$ is the group of all arrays

$$
\underline{c}=(\underline{c}(G / K)) \in \bigoplus_{K \leq G} \Gamma^{n}\left(X^{K} ; \underline{M}(G / K)\right)
$$

such that for any $n$-cell $E_{\alpha}^{n}=\left(E^{n} \stackrel{h_{\alpha}}{\rightarrow} X_{n}\right)$ of $X$ the equation

$$
\underline{c}(G / H)\left(g E_{\alpha}^{n}\right)=\underline{M}(\hat{g})\left(z_{\alpha}\right)\left(\underline{c}(G / K) E_{\alpha}^{n}\right)
$$

holds in $\underline{M}(G / K)\left(g z_{\alpha}\right)$ whenever $K \leq G$ fixes $E_{\alpha}^{n}$ and $g^{-1} H g \subset K$.

EXAMPLE 2.4. A. If each local coefficient system $\underline{M}(G / K)$ on $X^{K}$ is simple, then $\underline{M}: \mathscr{O}_{G} \rightarrow \mathscr{G}^{\text {ab }}$ is just an abelian $\mathscr{O}_{G}$-group and

$$
\Gamma_{G}^{n}(X ; \underline{\mathscr{M}})=\operatorname{Hom}_{\mathscr{O}_{G}}\left(\underline{\Gamma}_{n}(X), \underline{M}\right)
$$

reduces to the cellular $G$-cochains of Bredon [1].

B. Let $\pi_{i}(\mathscr{F}): \mathscr{O}_{G} \rightarrow \mathscr{L}$ be the system of Example 2.2 and $f: X \rightarrow B$ some equivariant map. Then the array

$$
\underline{c}=(\underline{c}(G / K)) \in \Gamma_{G}^{n}\left(X ; f^{*} \underline{\pi}_{i}(\mathscr{F})\right)
$$

if and only if

$$
\underline{c}(G / H)\left(g E_{\alpha}^{n}\right)=g_{*} \underline{c}(G / K)\left(E_{\alpha}^{n}\right) \in \pi_{i}\left(\left(p^{H}\right)^{-1}\left(g f z_{\alpha}\right)\right)
$$

whenever $K$ fixes $E_{\alpha}^{n}$ and $g^{-1} H g \subset K$.

We get a cochain complex $\left(\Gamma_{G}^{*}(X ; \mathscr{M}), \delta_{G}\right)$ simply by taking as $\delta_{G}^{n}$ the restriction to $\Gamma_{G}^{n}(X ; \mathscr{M})$ of the direct sum

$$
\underline{\delta}=\bigoplus \delta(G / K): \bigoplus \Gamma^{n}\left(X^{K} ; \underline{M}(G / K)\right) \rightarrow \bigoplus \Gamma^{n+1}\left(X^{K} ; \underline{M}(G / K)\right)
$$

of the ordinary coboundary operators. To do so, we of course first need to verify

LEMMA 2.5 .

$$
\underline{\delta}\left(\Gamma_{G}^{n}(X ; \underline{\mathscr{M}}) \subset \Gamma_{G}^{n+1}(X ; \underline{\mathscr{M}}) .\right.
$$

Proof. Suppose that $g^{-1} H g \subset K$ such that left multiplication

$$
\hat{g}: G / H \rightarrow G / K
$$

is a morphism in $\mathscr{O}_{G}$ and

$$
\underline{\mathscr{M}}(\hat{g})=(g, \underline{M}(\hat{g})):\left(X^{K}, \underline{M}(G / K)\right) \rightarrow\left(X^{H}, \underline{M}(G / H)\right)
$$


one in $\mathscr{L}$ inducing a commutative diagram

$$
\begin{array}{ccc}
\Gamma^{n}\left(X^{H} ; \underline{M}(G / H)\right) & \stackrel{\delta(G / H)}{\longrightarrow} & \Gamma^{n+1}\left(X^{H} ; \underline{M}(G / H)\right) \\
g^{*} \downarrow & \downarrow g^{*} \\
\Gamma^{n}\left(X^{K} ; g^{*} \underline{M}(G / K)\right) & \stackrel{\delta}{\longrightarrow} & \Gamma^{n+1}\left(X^{K} ; g^{*} \underline{M}(G / H)\right) \\
M(\hat{g}) * & \underline{M}(\hat{g}) * \\
\Gamma^{n}\left(X^{K} ; \underline{M}(G / K)\right) & \stackrel{\delta(G / K)}{\longrightarrow} & \Gamma^{n+1}\left(X^{K} ; \underline{M}(G / K)\right) .
\end{array}
$$

Suppose $\underline{c} \in \Gamma_{G}^{n}(X ; \underline{\mathscr{M}})$. The equations of Definition 2.3 are equivalent to the equations

$$
g^{*} \underline{c}(G / H)=\underline{M}(\hat{g})_{*} \underline{c}(G / K)
$$

and the commutative diagram above implies that $\underline{\delta c}$ satisfies these equations if $\underline{c}$ does so.

The above definition is easily generalized to relative $G-C W$ complexes $(X, A)$. For $G-C W$ pairs $(X, A)$ one then obtains a short exact sequence of cochain complexes

$$
0 \rightarrow \Gamma_{G}^{*}(X, A ; \underline{\mathscr{M}}) \rightarrow \Gamma_{G}^{*}(X ; \underline{\mathscr{M}}) \rightarrow \Gamma_{G}^{*}(A ; \underline{\mathscr{M}} \mid A) \rightarrow 0
$$

resulting in the long exact sequence for Bredon cohomology with local coefficients. Of course, this sequence is natural. (For this statement to be meaningful one must define morphisms in the category of local $G$-coefficient systems on $G-C W$ pairs; cf. ([20], p. 270).)

The scene is now set for equivariant obstruction theory. Suppose that $(X, A)$ is a relative $G-C W$ complex, $p: Y \rightarrow B$ a $G$-fibration as in Example 2.2, $f: X \rightarrow B$ a $G$-map, and

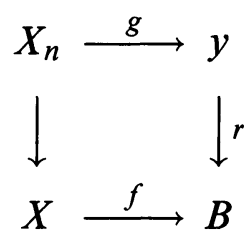

an equivariant lift of $f$ defined on the $n$-skeleton of $(X, A), n \geq 1$. It is routine to define an equivariant obstruction cycle

$$
c_{G}^{n+1}(g) \in \Gamma_{G}^{n+1}\left(X, A ; f^{*} \underline{\pi}_{n}(\mathscr{F})\right)
$$

to extending the lift $g$ equivariantly to the $(n+1)$-skeleton. In fact,

$$
c_{G}^{n+1}(g)=\left(c^{n+1}\left(g^{K}\right)\right) \in \bigoplus_{K \leq G} \Gamma^{n+1}\left(X^{K}, A^{K} ; f^{*} \underline{\pi}_{n}\left(\mathscr{F}^{K}\right)\right)
$$


where $c^{n+1}\left(g^{K}\right)$ is the usual non-equivariant obstruction to extending the lift $g^{K}: X_{n}^{K} \rightarrow Y^{K}$ of $f^{K}: X^{K} \rightarrow B^{K}$ to $X_{n+1}^{K}$. Example 2.4.B shows that $c_{G}^{n+1}(g)$ does satisfy the relations of Definition 2.3.

All the standard non-equivariant properties as listed in Whitehead ([20], VI) are easily transferred to the equivariant category. In particular, one may define equivariant primary obstruction and difference cohomology classes under suitable $G$-connectedness conditions. We shall return to this in the following chapter.

3. Realizing local $G$-coefficient systems. In this section we construct classifying fibrations for equivariant cohomology with local $G$ coefficients.

Let $(\mathscr{G}$ ab $) \mathscr{G}$ denote the category of (abelian) groups and let $\underline{\pi}: \mathscr{O}_{G} \rightarrow$ $\mathscr{G}$ and $\underline{M}: \mathscr{O}_{G} \rightarrow \mathscr{G}^{\text {ab }}$ be contravariant functors ( $\mathscr{O}_{G}$ groups for short).

Definition 3.1. A $\underline{\pi}$-module structure on $\underline{M}$ is a natural transformation $\underline{\pi} \times \underline{M} \rightarrow \underline{M}$ defining a $\underline{\pi}(G / K)$-module structure on $M(G / K)$ for each subgroup $K \leq G$.

If $B$ is a $G$-connected ([9], Definition 3) pointed $G$-space, then a $\underline{\pi}_{1}\left(B, b_{0}\right)$-module is the same thing as a local $G$-coefficient system on $B$ ([20], XV 1.11-1.12), so if $p: Y \rightarrow B$ is a $G$-fibration with $G$ connected and $G$-simple fibre $F=p^{-1}\left(b_{0}\right)$, then $\underline{\pi}_{i}(F)$ is a $\underline{\pi}_{1}\left(B, b_{0}\right)$ module for all $i \geq 1$. The next lemma shows, conversely, that in fact any $\pi$-module $\underline{M}$ has such a geometric realization.

Recall that if $\underline{M}$ is an (abelian) $\mathscr{O}_{G}$-group and $n \geq 1$ an integer, then $K(\underline{M}, n)$ denotes any $G$-connected pointed $G$-space $G$-homotopy equivalent to a $G-C W$ complex with $\underline{\pi}_{n} K(\underline{M}, n)=\underline{M}$ and $\underline{\pi}_{i} K(\underline{M}, n)$ $=0$ for $i \neq n$. See [2], [18] for the existence of these equivariant Eilenberg-Mac Lane spaces.

LeMma 3.2. Let $\underline{M}$ be a $\underline{\pi}$-module and $n \geq 1$ an integer. There exists a sectioned $G$-fibration

$$
K(\underline{M}, n) \rightarrow L(\underline{\pi}, \underline{M}, n) \underset{s}{\stackrel{p}{\rightleftarrows}} K(\underline{\pi}, 1)
$$

of G-connected pointed G-spaces G-homotopy equivalent to $G-C W$ complexes realizing the given module structure as the associated action of $\underline{\pi}_{1} K(\underline{\pi}, 1)=\underline{\pi}$ on $\underline{\pi}_{n} K(\underline{M}, n)=\underline{M}$.

Proof. Let $E, B: \mathscr{G} \rightarrow$ Top be Milnor's functors for the construction of universal principal bundles [7] and let $E \underline{\pi}, B \underline{\pi}: \mathscr{O}_{G} \rightarrow$ Top be the $\mathscr{O}_{G}$-spaces [2] obtained by pre-composition with $\underline{\pi}$. 
For each subgroup $K \leq G$, let $B^{n} \underline{M}(G / K)$ denote (the geometric realization of) the iterated bar construction on $\underline{M}(G / K)$; cf. ([2], §2). The $\underline{\pi}(G / K)$-module structure on $M(G / K)$ determines a representation

$$
\varphi(G / K): \underline{\pi}(G / K) \rightarrow \operatorname{Aut}_{0}\left(B^{n} \underline{M}(G / K)\right)
$$

of $\underline{\pi}(G / K)$ as a group of based homeomorphisms of $B^{n} \underline{M}(G / K)$ such that $([11], \S 3)$, for any $\xi \in \underline{\pi}(G / K), \varphi(G / K)(\xi)_{*}$ is multiplication by $\xi$ on $\pi_{n}\left(B^{n} \underline{M}(G / K)\right)=\underline{M}(G / K)$. Since $\underline{M}$ is a $\underline{\pi}$-module, these homeomorphisms behave coherently in the sense that

$$
B^{n} \underline{M}(\hat{g}) \circ \underline{\varphi}(G / K)(\xi)=\varphi(G / H)(\underline{\pi}(\hat{g}) \xi) \circ B^{n} \underline{M}(\hat{g})
$$

for any $\mathscr{O}_{G}$-morphism $\hat{g}: G / H \rightarrow G / K$. It follows that there is a well defined $\mathscr{O}_{G}$-space, $l(\underline{\pi}, \underline{\mathscr{M}}, n)$, which on $G / K \in \mathscr{O}_{G}$ is given by

$$
l(\underline{\pi}, \underline{M}, n)(G / K)=E \underline{\pi}(G / K) \times_{\underline{\pi}(G / K)} B^{n} \underline{M}(G / K) .
$$

Note that this space is the total space of a sectioned fibration

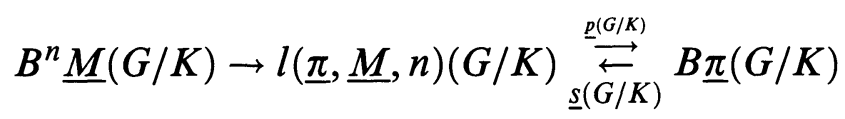

from which the $\underline{\pi}(G / K)$-module structure on $\underline{M}(G / K)$ can be recovered as the action of $\pi_{1}$ (base) on $\pi_{n}$ (fibre). The collection of these sectioned fibrations constitute a diagram

$$
l(\underline{\pi}, \underline{M}, n) \underset{\underline{s}}{\stackrel{\underline{p}}{\rightleftarrows}} B \underline{\pi}
$$

in the category of $\mathscr{O}_{G}$ spaces. Apply Elmendorf's functor $C$ to it [2]:

$$
C l(\underline{\pi}, \underline{M}, n) \underset{C \underline{\underline{s}}}{\stackrel{C \underline{p}}{\rightleftarrows}} C B \underline{\pi}=K(\underline{\pi}, 1) .
$$

$C p$ is in general just a quasi-fibration [8], [12]. At each fixed point set there exist, however, homotopy equivalences ([2], Theorem 1) such that the diagram

$$
\begin{array}{ccc}
C l(\underline{\pi}, \underline{M}, n)^{K} & \stackrel{\eta}{\cong} & l(\underline{\pi}, \underline{M}, n)(G / K) \\
(C \underline{p})^{K} \downarrow \uparrow(C \underline{s})^{K} & & \downarrow \uparrow \\
(B \underline{\pi})^{K} & \stackrel{\eta}{\cong} & B \underline{\pi}(G / K)
\end{array}
$$

commutes. Hence the fibre of $C \underline{p}$ has only one non-trivial $\mathscr{O}_{G}$-homotopy group namely

$$
\underline{\pi}_{n}=\underline{\pi}_{n+1}(K(\underline{\pi}, 1), C l(\underline{\pi}, \underline{M}, n))=\underline{M},
$$


and the induced natural transformation

$$
\begin{aligned}
\underline{\pi} \times \underline{M} & =\underline{\pi}_{1}(K(\underline{\pi}, 1)) \times \underline{\pi}_{n+1}(K(\underline{\pi}, 1), C l(\underline{\pi}, \underline{M}, n)) \\
& \left(\stackrel{\underline{s}}{)} \times 1_{\longrightarrow} \underline{\pi}_{1}(C l(\underline{\pi}, \underline{M}, n)) \times \underline{\pi}_{n+1}(K(\underline{\pi}, 1), C l(\underline{\pi}, \underline{M}, n))\right. \\
& \rightarrow \underline{\pi}_{n+1}(K(\underline{\pi}, 1), C l(\underline{\pi}, \underline{M}, n))=\underline{M}
\end{aligned}
$$

is the presentation of $\underline{M}$ as a $\underline{\pi}$-module.

Now factor $C \underline{p}$ as a $G$-homotopy equivalence, $u$, followed by a $G$ fibration, $p$,

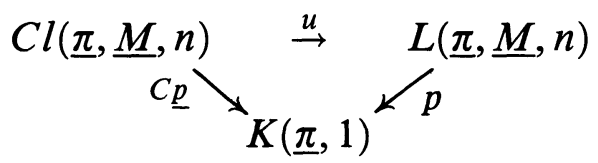

This doesn't change the associated action of the base space on the fibre. Moreover, the fibre of $p$, i.e. the $G$-homotopy fibre of $C p$, is a $K(\underline{M}, n)$ since it has the $G$-homotopy type of a $G-C W$-complex ([19], Corollary 4.14) and only one non-trivial homotopy $\mathscr{O}_{G}$-group.

Finally, put $s=u \circ C \underline{s}$. Then $p s=p u \circ C s=C \underline{p} \circ C \underline{s}=C(\underline{p} \circ \underline{s})=$ $C(\mathrm{id})=\mathrm{id}$, by functoriality of $C$.

Suppose that $u: X \rightarrow L(\underline{\pi}, \underline{M}, n)$ is a $G$-map on a relative $G-C W$ complex $(X, A)$. Put $u_{1}=p u$. Consider the space

$$
F_{u}(X, A ; L(\underline{\pi}, \underline{M}, n), K(\underline{\pi}, 1))^{G}
$$

of all $G$-maps $v: X \rightarrow L(\underline{\pi}, \underline{M}, n)$ such that $p v=p u$ and $u|A=v| A$. Associated to any such $v$ is a primary obstruction

$$
\delta_{G}^{n}(u, v) \in H_{G}^{n}\left(X, A ; u_{1}^{*} \underline{\mathscr{M}}\right)
$$

to $G$-homotoping $u$ vertically (rel. $A$ ) to $v$; here $\underline{\mathscr{M}}$ is $\underline{M}$ considered as a local $G$-coefficient system on $K(\underline{\pi}, 1)$. Obstruction theory yields

THEOREM 3.3. There is a bijection

$$
\pi_{0} F_{u}(X, A ; L(\underline{\pi}, \underline{M}, n), K(\underline{\pi}, 1)) \rightarrow H_{G}^{n}\left(X, A ; u_{1}^{*} \underline{\mathscr{M}}\right)
$$

induced by the map $v \rightarrow \delta_{G}^{n}(u, v)$.

It is for this reason that the $G$-fibration of Lemma 3.2 deserves to be called the classifying fibration for equivariant Bredon cohomology with local $G$-coefficients.

Over $L(\underline{\pi}, \underline{M}, n)$ is another fundamental $G$-fibration

$$
K(\underline{M}, n-1) \rightarrow \bar{P} L(\underline{\pi}, \underline{M}, n) \rightarrow L(\underline{\pi}, \underline{M}, n)
$$


which, in analogy with [11], is called the equivariant path fibration over and under $K(\underline{\pi}, 1)$ and which is constructed by factoring the section $s$

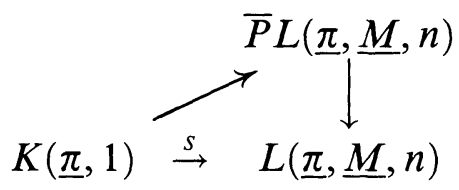

into a $G$-homotopy equivalence followed by a $G$-fibration. This $G$ fibration will later serve as a typical building block in equivariant Moore-Postnikov factorizations. As preparation for the construction of these factorizations we now continue to list a few further properties of $L(\underline{\pi}, \underline{M}, n)$.

Now suppose that both $\underline{L}$ and $\underline{M}$ are $\underline{\pi}$-modules and let $\operatorname{Hom}_{\underline{\pi}}(\underline{L}, \underline{M})$ be the abelian group of natural $\underline{\pi}$-module transformations of $\underline{L}$ into $\underline{M}$. This functor $C$ of [2] induces a map

$$
\operatorname{Hom}_{\underline{\pi}}(\underline{L}, \underline{M}) \rightarrow\langle L(\underline{\pi}, \underline{L}, n), L(\underline{\pi}, \underline{M}, n)\rangle_{K(\underline{\pi}, 1)}^{G}
$$

into the set of $G$-homotopy classes of $G$-maps over and under $K(\underline{\pi}, 1)$. An inverse is obtained by associating to each $G$-map $u$ over and under $K(\underline{\pi}, 1)$ the induced map

$$
u_{*}: \underline{L}=\underline{\pi}_{n}(L(\underline{\pi}, \underline{L}, n), K(\underline{\pi}, 1)) \rightarrow \underline{\pi}_{n}(L(\underline{\pi}, \underline{M}, n), K(\underline{\pi}, 1))=\underline{M}
$$

of $\underline{\pi}_{n}(K(\underline{\pi}, 1))=\underline{\pi}$-modules. This proves an equivariant version of ([14]), Lemma 2.1).

LEMMA 3.4. There is a bijective correspondence

$$
\operatorname{Hom}_{\underline{\pi}}(\underline{L}, \underline{M}) \leftrightarrow\langle L(\underline{\pi}, \underline{L}, n), L(\underline{\pi}, \underline{M}, n)\rangle_{K(\underline{\pi}, 1)}^{G} .
$$

COROllary 3.5 .

$$
H_{G}^{n}\left(L(\underline{\pi}, \underline{L}, n), K(\underline{\pi}, 1) ; p^{*} \underline{\mathscr{M}}\right)=\operatorname{Hom}_{\underline{\pi}}(\underline{L}, \underline{M}) .
$$

COROLlARY 3.6. There is a short split-exact sequence $0 \rightarrow H_{G}^{n}(K(\underline{\pi}, 1) ; \underline{\mathscr{M}}) \underset{s^{*}}{\stackrel{p^{*}}{\rightleftarrows}} H^{n}\left(L(\underline{\pi}, \underline{M}, n) ; p^{*} \underline{\mathscr{M}}\right) \stackrel{i^{*}}{\rightarrow} \operatorname{Hom}_{\underline{\pi}}(\underline{M}, \underline{M}) \rightarrow 0$ 
Proof. Chase the commutative diagram:

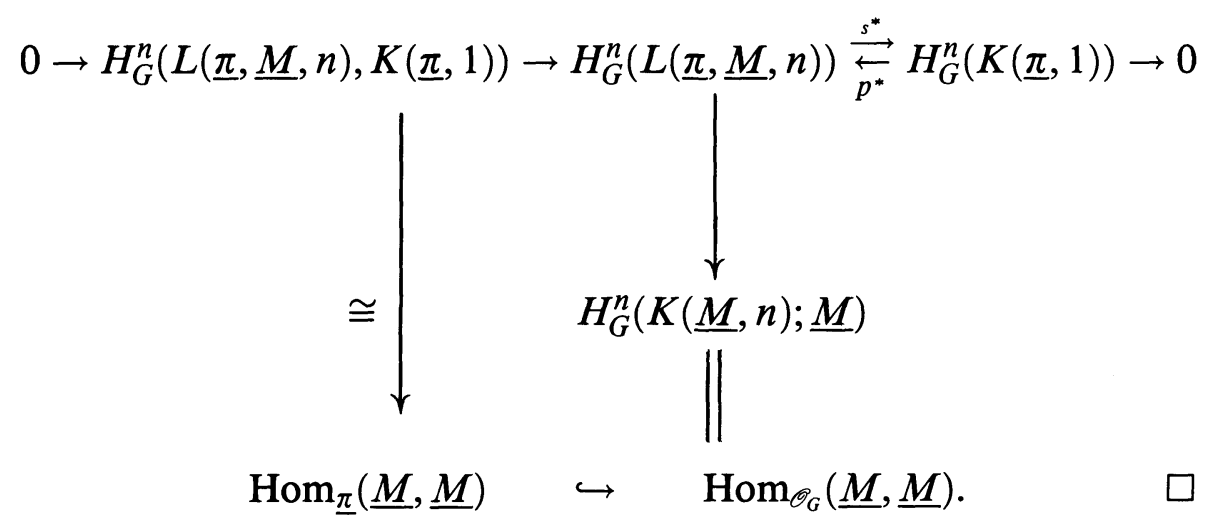

The cohomology classes

$$
\delta_{G}^{n}(\underline{M}), \gamma_{G}^{n}(\underline{M}) \in H_{G}^{n}\left(L(\underline{\pi}, \underline{M}, n) ; p^{*} \mathscr{M}\right)
$$

are defined, respectively, as the primary $G$-difference $\delta_{G}^{n}(\underline{M})=\delta^{n}(1, s p)$ of the lifts 1 and $s p$ over $p$ and the primary obstruction to sectioning the equivariant path-space fibration $\bar{P} L(\underline{\pi}, \underline{M}, n) \rightarrow L(\underline{\pi}, \underline{M}, n)$.

Corollary 3.7. $\delta_{G}(\underline{M})=\gamma_{G}(\underline{M})$.

Proof. This follows from the short split-exact sequence of Corollary 3.6 since both cohomology classes are mapped to zero by $s^{*}$ and to the identity transformation by $i^{*}$.

4. Equivariant Postnikov resolutions. Equivariant Postnikov resolutions of $G$-spaces have been constructed by Triantafillou [18] and Elmendorf [2]. We shall here develop a theory, following [11], [17] for equivariant resolutions of $G$-fibrations.

Consider a $G$-fibration $p: Y \rightarrow B$ with base points $y_{0} \in Y^{G}$ and $b_{0}=p\left(y_{0}\right) \in B^{G}$. Assume that all three spaces $F=p^{-1}\left(b_{0}\right), Y$ and $B$ are $G$-connected and $G$-homotopy equivalent to $G-C W$ complexes. Put $\underline{\pi}=\underline{\pi}_{1}\left(B, b_{0}\right)$.

Let $\underline{M}$ be an abelian $\mathscr{O}_{G}$-group and

$$
[F, K(\underline{M}, n)]_{G}=\operatorname{Hom}_{\mathscr{O}_{G}}\left(\underline{\pi}_{n}\left(F, y_{0}\right), \underline{M}\right)
$$

the set of based $G$-homotopy classes of $G$-maps of $F$ into $K(\underline{M}, n)$ identified ([1], I-26) to the corresponding set of natural transformation. 
If, furthermore, $\underline{M}$ is equipped with a $\underline{\pi}$-module structure, as we shall assume from now on, then the classifying $G$-fibration

$$
L(\underline{\pi}, \underline{M}, n+1) \rightarrow K(\underline{\pi}, 1)
$$

exists. Make also $B$ into a $G$-space by choosing a based $G$-map $B \rightarrow$ $K(\underline{\pi}, 1)$ inducing the identity on fundamental $\mathscr{O}_{G}$-groups.

Definition 4.1. We say that the $G$-map $\alpha: F \rightarrow K(\underline{M}, n), n \geq 1$, can be $G$-realized by the $G$-map $k: B \rightarrow L(\underline{\pi}, \underline{M}, n+1)$ over $K(\underline{\pi}, 1)$ if $k$ lifts to a $G$-fibre map

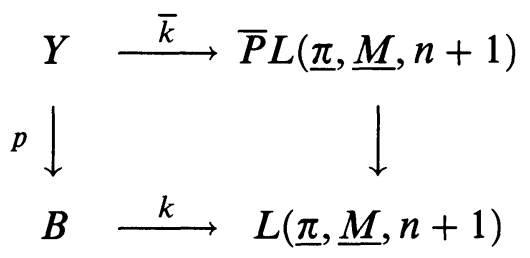

such that $\bar{k} \mid F: F \rightarrow K(\underline{M}, n)$ is $G$-homotopic to $\alpha$.

All maps and homotopies in this definition are assumed to be based. Hence $\alpha$ and $k$ represent cohomology classes in $\widetilde{H}_{G}^{n}(F ; \underline{M})$ and $\widetilde{H}_{G}^{n+1}(B ; \underline{M})$, respectively. If $F$ is $G-(n-1)$-connected, then here is a partially defined homomorphism connecting these two Bredon cohomology groups:

Definition 4.2. Suppose that $F$ is $G-(n-1)$-connected (and that $\underline{\pi}_{1}(F)$ is abelian if $\left.n=1\right)$ and let $\gamma_{G}^{n+1}(p) \in \widetilde{H}_{G}^{n+1}\left(B ; \underline{\pi}_{n}(\mathscr{F})\right)$ be the primary obstruction to sectioning $p$. The homomorphism

$$
\begin{aligned}
\tau_{G}: \operatorname{Hom}_{\underline{\pi}}\left(\underline{\pi}_{n}(F), \underline{M}\right) & \rightarrow \tilde{H}_{G}^{n+1}(B ; \underline{\mathscr{M}}) \\
\alpha & \rightarrow \alpha_{*} \gamma_{G}^{n+1}(p)
\end{aligned}
$$

is called the equivariant transgression.

With the help of these concepts we can now formulate an equivariant version of ([11], Theorem 4.1). The assumptions of $F$ are as in Definition 4.2.

Theorem 4.3. Any G-map $F \rightarrow K(\underline{M}, n)$ in the G-homotopy class of an

$$
\left.a \in \operatorname{Hom}_{\underline{\pi}}(F), \underline{M}\right) \subset \widetilde{H}_{G}^{n}(f ; \underline{M})
$$

can be G-realized by a G-map over $K(\underline{\pi}, 1)$ in the G-homotopy class of $\tau_{G}(\alpha) \in \widetilde{H}_{G}^{n+1}(B ; \underline{M})$. 
The proof of this theorem follows the scheme of [11] and is accordingly omitted. I needed Corollary 3.7 to prove the $G$-version of ([11], Lemma 2.1).

We are now in a position to copy [14] and factor $p: Y \rightarrow B$ into equivariant fibrations of the following type:

Definition 4.4. Let $Z \rightarrow K(\underline{\pi}, 1)$ be a $G$-space over $K(\underline{\pi}, 1)$. Any equivariant fibration over $Z$ obtained as the pullback of a diagram of the form

$$
\begin{gathered}
\bar{P} L(\underline{\pi}, \underline{M}, n+1) \\
\downarrow \\
Z \stackrel{k}{\longrightarrow} L(\underline{\pi}, \underline{M}, n+1)
\end{gathered}
$$

where $n \geq 1, \underline{M}$ is some $\underline{\pi}$-module, and $k$ some $G$-map over $K(\underline{\pi}, 1)$, is called a $K(\underline{\pi}, 1)$-principal $G$-fibration.

Suppose that

$$
0 \rightarrow \underline{A} \stackrel{\kappa}{\rightarrow} \underline{B} \rightarrow \underline{\pi} \rightarrow 1
$$

is an exact sequence of $\mathscr{O}_{G}$-groups and that $\underline{N}$ is a $\underline{B}$-module. Consider $\underline{N}$ as an $\underline{A}$-module through $\kappa$ and form the descending chain of abelian $\mathscr{O}_{G}$-groups

$$
\underline{N}=\Gamma_{\underline{A}}^{1}(\underline{N}) \supset \Gamma_{\underline{A}}^{2}(\underline{N}) \supset \cdots \supset \Gamma_{\underline{A}}^{i}(\underline{N}) \supset \Gamma_{\underline{A}}^{i}(\underline{N}) \supset \cdots
$$

where $\Gamma_{A}^{i+1}(\underline{N})(G / H)$ is the subgroup of $\Gamma_{A}^{i}(\underline{N})(G / H)$ generated by the set $\{a m-m \mid a \in \underline{A}(G / H), m \in \underline{N}(G / \bar{H})\}$. Note ([14], Lemma 3.2) that each $\Gamma_{\underline{A}}^{i}(\underline{N})$ is a $\underline{G}$-submodule of $\underline{N}$ and all subquotients $\Gamma_{\underline{A}}^{i}(\underline{N}) / \Gamma_{\underline{A}}^{i+1}(\underline{N})$, being trivial $\underline{A}$-modules, inherit a $\underline{\pi}$-module structure. The equivariant path fibrations over $K(\underline{\pi}, 1)$

$$
\bar{P} L\left(\underline{\pi}, \Gamma_{\underline{A}}^{i}(\underline{N}) / \Gamma_{\underline{A}}^{i+1}(\underline{N}), n+1\right) \rightarrow L\left(\underline{\pi}, \Gamma_{\underline{A}}^{i}(\underline{N}) / \Gamma_{\underline{A}}^{i+1}(\underline{N}), n+1\right)
$$

then exist. Using Lemma 3.7 and Theorem 4.3 these $G$-fibrations can be exploited to prove the following equivariant version of ([14], Lemma 3.3).

LemMA 4.5. Suppose that $\underline{N}$ is $\underline{A}$-nilpotent (i.e. that $\Gamma_{\underline{A}}^{c+1}(\underline{N})=0$ for some $c \geq 1)$. Then the equivariant path fibration over $\underline{K(} \underline{B}, 1)$

$$
K(\underline{N}, n) \rightarrow \bar{P} L(\underline{B}, \underline{N}, n+1) \rightarrow L(\underline{B}, \underline{N}, n+1)
$$

can be factored into a finite string of $K(\underline{\pi}, 1)$-principal $G$-fibrations.

It is understood that $K(\underline{B}, 1)$, and then also $L(\underline{B}, \underline{N}, n+1)$, is a $G$-space over $K(\underline{\pi}, 1)$ through the projection of $\underline{B}$ onto $\underline{\pi}$. 
The equivariant version of ([14], Theorem 3.4) is now readily obtained.

TheORem 4.6. Suppose that $F$ is $G$-nilpotent and that $\underline{\pi}_{1}(F)$ is abelian. Then, for any given $\gamma \geq 1$, there exists a factorization

$$
Y \rightarrow Y_{s+1} \rightarrow \cdots \rightarrow Y_{i+1} \rightarrow Y_{i} \rightarrow \cdots \rightarrow Y_{2} \rightarrow Y_{1}=B
$$

of $p: y \rightarrow B$ into a finite string of equivariant fibrations such that $Y \rightarrow$ $Y_{s+1}$ is $G-(r+1)$-connected and each $Y_{i+1} \rightarrow Y_{i}, 1 \leq i \leq s$, is a $K(\underline{\pi}, 1)$-principal $G$-fibration.

Proof. First $G$-realize the identity map of $\underline{\pi}_{1}(F)$ as in Theorem 4.3. There results a factorization

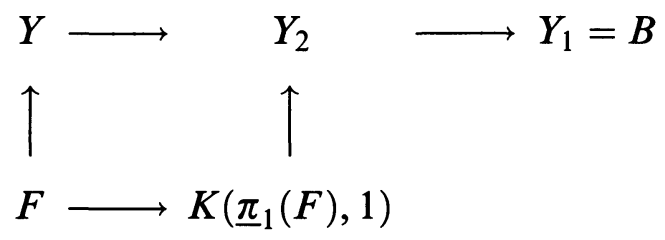

of $p$ such that $G$-homotopy fibre $Y \rightarrow Y_{2}$ is $G$-simply connected, in particular $G$-simple. Then (Theorem 4.3 again) factor $Y \rightarrow Y_{2}$ into a finite string of $K\left(\underline{\pi}_{1}\left(Y_{2}\right), 1\right)$-principal $G$-fibrations in the usual way by killing the homotopy groups of the fibre one at a time. Finally apply Lemma 4.5 with $\underline{A}=\underline{\pi}_{1}(F), \underline{B}=\underline{\pi}_{1}\left(Y_{2}\right)=\underline{\pi}_{1}(Y), \underline{N}=\underline{\pi}_{n}(F)=$ $\underline{\pi}_{n+1}(B, Y), n>1$, and the $G$-nilpotency of $F$ to factor each of these $K\left(\underline{\pi}_{1}(Y), 1\right)$-principal fibrations into finitely many $K(\underline{\pi}, 1)$-principal $G$-fibrations.

The condition that $\underline{\pi}_{1}(F)$ be abelian seems to be of a technical nature and can presumably be omitted.

5. Applications to equivariant mapping spaces. Let $p: Y \rightarrow B$ be a $G$-fibration as in the previous section, let $(X, A)$ be a relative $G-C W$ complex, and let $u: X \rightarrow Y$ be a $G$-map. We shall study the space $F_{u}(X, A ; Y, B)^{G}$, consisting of all equivariant maps $v: X \rightarrow Y$ such that $v|A=u| A$ and $p v=p u=: u_{1}$, under various assumptions on the fibre $F$.

Suppose first that $\underline{M}: \mathscr{O}_{G} \rightarrow \mathscr{G}^{\text {ab }}$ is an abelian $\mathscr{O}_{G}$-group and $F=$ $K(\underline{M}, n), n \geq 1$, is the corresponding equivariant Eilenberg-Mac Lane complex. Let $\underline{\mathscr{M}}=\underline{\pi}_{n}(\mathscr{F})$ denote the resulting local $G$-coefficient system on $B$. 
THEOREM 5.1. There exists a weak homotopy equivalence

$$
F_{u}(X, A ; Y, B)^{G} \rightarrow \prod_{i+j=n} K\left(H_{G}^{j}\left(X, A ; u_{1}^{*} \underline{\mathscr{M}}\right), i\right)
$$

which is natural in the first argument.

Theorem 5.1 follows, exactly as in [13], from a Künneth splitting:

LEMMA 5.2. There exists an isomorphism of cofunctors

$$
H_{G}^{*}\left(? \times(X, A) ; p r_{2}^{*} \underline{\mathscr{M}}\right) \rightarrow H^{*}\left(? ; H_{G}^{*}(X, A ; \underline{\mathscr{M}})\right)
$$

on the category of trivial $G-C W$ complexes.

Proof. Let $Z$ be a trivial $G-C W$ complex (i.e. $Z^{G}=Z$ ) and let $\left(\Gamma^{*}\right) \Gamma_{*}$ denote the cellular (co)chain complex functor.

For each subgroup $H \leq G$, there is a natural adjointness isomorphism of chain complexes,

$\Gamma^{*}\left(Z \times\left(X^{H}, A^{H}\right) ; p r_{2}^{*} \underline{\mathscr{M}}(G / H)\right) \cong \operatorname{Hom}\left(\Gamma_{*}(Z), \Gamma^{*}\left(X^{H}, A^{H} ; \underline{\mathscr{M}}(G / H)\right)\right)$

and the collection of all these form an isomorphism

$$
\Gamma_{G}^{*}\left(Z \times(X, A) ; p r_{2}^{*} \underline{\mathscr{M}}\right) \cong \operatorname{Hom}\left(\Gamma_{*}(Z), \Gamma_{G}^{*}(X, A ; \underline{\mathscr{M}})\right) .
$$

Compose this isomorphism with the quasi-isomorphism

$$
\operatorname{Hom}\left(?, \Gamma_{G}^{*}(X, A ; \underline{\mathscr{M}})\right) \rightarrow \operatorname{Hom}\left(?, H_{G}^{*}(X, A ; \underline{\mathscr{M}})\right)
$$

of ([13], Lemma 2.2).

Suppose next that $F$ is $G$-simple ([19], Definition 3) such that the $\underline{\pi}$-module $\underline{\pi}_{*}(F)$ or, alternatively, the local $G$-coefficient system $\underline{\pi}_{*}(\mathscr{F})$ is defined. Then, by Theorem 4.6, we can factor $p: Y \rightarrow B$ into finite strings

$$
\begin{gathered}
Y \rightarrow Y_{r+1} \rightarrow \cdots \rightarrow \underset{\uparrow}{Y_{i+1}} \rightarrow Y_{i} \rightarrow \cdots \rightarrow Y_{2} \rightarrow Y_{1}=B \\
K\left(\underline{\pi}_{i}(F), i\right)
\end{gathered}
$$

of $K(\underline{\pi}, 1)$-principal $G$-fibrations inducing finite strings

$$
\begin{gathered}
F_{u}(X, A ; Y, B)^{G} \rightarrow \cdots \rightarrow F_{u}\left(X, A ; Y_{i+1}, B\right)^{G} \rightarrow F_{u}\left(X, A ; Y_{i}, B\right)^{G} \rightarrow \cdots \\
\uparrow \\
\prod_{\alpha+\beta=i} K\left(H_{G}^{\beta}\left(X, A ; \underline{\pi}_{i}(\mathscr{F})\right), \alpha\right)
\end{gathered}
$$


of fibrations of equivariant mapping spaces. The interlocking homotopy sequences for these fibrations constitute an exact couple

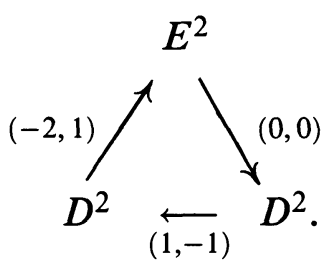

with homomorphisms of the indicated bidegrees and with

$$
\begin{aligned}
& E_{p q}^{2}=\pi_{p+q}\left(F_{u}\left(X, A ; Y_{q+1}, Y_{q}\right)^{G}, u\right), \\
& D_{p q}^{2}=\pi_{p+q}\left(F_{u}\left(X, A ; Y_{q+1}, B\right)^{G}, u\right) .
\end{aligned}
$$

Since

$$
E_{p q}^{2}= \begin{cases}H_{G}^{-p}\left(X, A ; \underline{\pi}_{q}(\mathscr{F})\right), & q \geq 0, p+q \geq 0, \\ 0 & \text { otherwise }\end{cases}
$$

by Theorem 5.1, this exact couple generates a Federer spectral sequence [3], [15], convergent under certain finiteness conditions.

TheORem 5.3. Suppose that $F$ is $G$-simple and that $(X, A)$ is finite dimensional or that $F$ is finitely $G$-anticonnected. Then there exists a 2nd quadrant homology spectral sequence with

$$
E_{p q}^{2}=H_{G}^{-p}\left(X, A ; \underline{\pi}_{q}(\mathscr{F})\right)
$$

for $p+q \geq 0$ and $E_{p q}^{2}=0$ otherwise, converging to

$$
\pi_{p+q}\left(F_{u}(X, A ; Y, B)^{G}, u\right)
$$

when $p+q>0$.

EXAMPLE 5.4. Let $G=\mathrm{Z} / 2$ act on $\left(S^{n}, *\right), n>2$, by reflection in a hyperplane. Then $\pi_{*+n}\left(F_{u}\left(S^{n}, * ; Y\right)^{G}\right)=\pi_{*+n}\left(Y, Y^{G}\right)$ for any $G$-simple space $Y$. The above spectral sequence has

$$
E_{p q}^{2}= \begin{cases}\operatorname{coker}\left(\pi_{q}\left(Y^{G}\right) \rightarrow \pi_{q}(Y)\right), & p=-n, q \geq n, \\ \operatorname{ker}\left(\pi_{q}\left(Y^{G}\right) \rightarrow \pi_{q}(Y)\right), & p=-n+1, q \geq n-1, \\ 0, & \text { otherwise. }\end{cases}
$$

and $d_{2}=0$. The spectral sequence of R. Schultz, ([15], Theorem II.4.4) with $\pi_{p+q+i}\left(Y^{i-p}\right)$ corrected to $\pi_{p+q-i}\left(Y^{i-p}\right)$, has

$$
E_{p q}^{2}= \begin{cases}\pi_{q-2}(Y), & p=-n-2 \\ \pi_{q-1}\left(Y^{G}\right), & p=-n \\ 0, & \text { otherwise }\end{cases}
$$


and the differential

$$
d^{2}: \pi_{q}\left(Y^{G}\right)={ }_{S} E_{-n, q+1}^{2} \rightarrow{ }_{S} E_{-n-2, q+2}^{2}=\pi_{q}(Y)
$$

is induced by the inclusion. Thus ${ }_{S} E^{3} \cong E^{2}$ and we see that these two spectral sequences, in cases where they both apply, are not in general isomorphic.

As to the global structure of $F_{u}(X, A ; Y, B)^{G}$ one has

THEOREM 5.5. Suppose that $F$ is G-nilpotent with abelian fundamental $\mathscr{O}_{G}$-group and that the finiteness condition of Theorem 5.3 is satisfied. Then each component of $F_{u}(X, A ; Y, B)^{G}$ is nilpotent.

To prove this statement, apply the refined Postnikov tower of Theorem 4.5 and proceed as in [14].

A convenient feature of nilpotent spaces is the existence of localizations and we shall next determine a localization of the component $F_{u}^{0}(X, A ; Y, B)^{G}$ of $F_{u}(X, A ; Y, B)^{G}$ containing $u$.

Let $\underline{\mathscr{M}}: \mathscr{O}_{G} \rightarrow \mathscr{L}$ be a local $G$-coefficient system on the relative $G-$ $C W$ complex $(X, A)$, let $P$ be a family of primes, and let $\mathscr{M}_{P}: \mathscr{O}_{G} \rightarrow \mathscr{L}$ be the $P$-localization of $\mathscr{M}$ defined in the obvious way: If $\mathscr{M}(G / H)=$ $\left(X^{H}, \underline{\mathscr{M}}(G / H)\right)$ the $\underline{\mathscr{M}}_{P}(G / H)=\left(X^{H}, \underline{\mathscr{M}}(G / H)_{P}\right)$. We denote the $P$ localization morphism by $e: \underline{\mathscr{M}} \rightarrow \underline{\mathscr{M}}_{P}$.

LEMMA 5.6. The coefficient group homomorphism

$$
e_{*}: H_{G}^{*}(X, A ; \underline{\mathscr{M}}) \rightarrow H_{G}^{*}\left(X, A ; \underline{\mathscr{M}}_{P}\right)
$$

is a P-localization if $(X, A)$ has finite skeleta.

Proof. For each $n \geq 0$ and each configuration $(H, g, K)$ such that $g^{-1} H g \subset K$, let $\underline{\mathscr{M}}(H, g, K)$ denote the equalizer of the homomorphisms

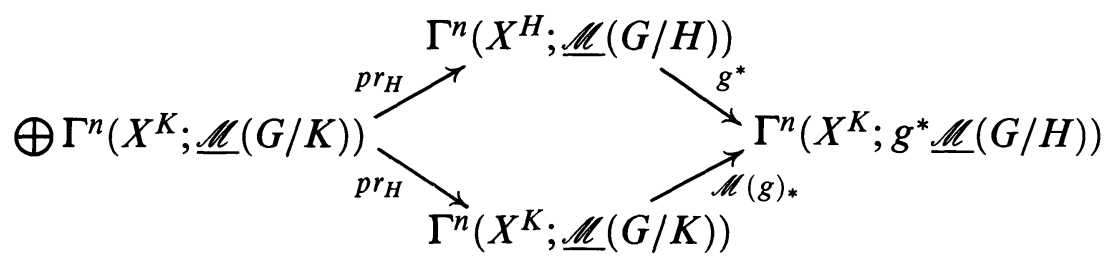

Then $\Gamma_{G}(X, A ; \underline{\mathscr{M}})=\bigcap \mathscr{M}(H, g, K)$ is the intersection of these finitely many equilizer subgroups and since, moreover,

$$
e_{*}: \Gamma^{n}(X, A ; \underline{\mathscr{M}}(G / H)) \rightarrow \Gamma^{n}\left(X, A ; \underline{\mathscr{M}}(G / H)_{P}\right)
$$


is a $P$-localization ([14], Lemma 5.1), the lemma follows from the general facts that localization of abelian groups is an exact functor which commutes with direct sums, equalizers, and finite intersections [4], [6].

In [9], J. P. May et al. proved the existence of an equivariant $P$ localization $e: F \rightarrow F_{P}$ for the $G$-nilpotent $G$-space $F$. Suppose that $Y_{(P)} \rightarrow B$ is a $G$-fibration (of the type considered here) with fibre $F_{P}$ and that $e$ extends to an equivariant fibre map $e: Y \rightarrow Y_{(P)}$ over $B$. Let

$$
\underline{e}: F_{u}^{0}(X, A ; Y, B)^{G} \rightarrow F_{e u}^{0}\left(X, A ; Y_{(P)}, B\right)^{G}
$$

be the map defined by post-composition with this map $e$.

THEOREM 5.7. Suppose in addition to the assumptions of Theorem 5.4 that $(X, A)$ has finite skeleta. Then $\underline{e}$ is a P-localization.

Proof. Replace ([14], Lemma 5.2) by Lemma 5.5 but proceed otherwise as in [14], [5] by induction on the refined Postnikov tower of Theorem 4.6.

See [4], [5], [21] for non-equivariant versions of 5.5 and 5.7.

Finally, let me use the opportunity for a correction. Corollary 5.4 of [14] is incorrectly stated as the nonequivariant localization of $F$ need not be $G$-space. Instead, the right hand side of the equality should be a component of the section space of $\left(\bar{X} \times_{G} F\right)_{(P)} \rightarrow X$.

\section{REFERENCES}

[1] G. E. Bredon, Equivariant Cohomology Theories, Lecture Notes in Mathematics 34, Springer-Verlag, Berlin, Heidelberg, New York 1967.

[2] A. D. Elmendorf, Systems of fixed point sets, Trans. Amer. Math. Soc., 277 (1983), 275-284.

[3] H. Federer, A study of function spaces by spectral sequences, Trans. Amer. Math. Soc., 82 (1956), 340-361.

[4] P. Hilton, G. Mislin and J. Roitberg, Localization of Nilpotent Groups and Spaces, North-Holland Publishing Company, Amsterdam 1975.

[5] P. Hilton, G. Mislin, J. Roitberg, and R. Steiner, On free maps and free homotopies into nilpotent spaces, In: Algebraic Topology, Proceedings, Vancouver 1977. Lecture notes in Mathematics 673, Springer-Verlag, Berlin, Heidelberg, New York 1978.

[6] P. Hilton, Nilpotente Gruppen und Nilpotente Raume, Lecture Notes in Mathematics 1053, Springer-Verlag, Berlin, Heidelberg, New York, Tokyo 1984.

[7] D. Husemoller, Fibre Bundles, Second Edition. Graduate Texts in Mathematics 20, Springer-Verlag, New York, Heidelberg, Berlin 1975. 
[8] J. P. May, Classifying spaces and fibrations, Mem. Amer. Math. Soc., 155 (1975).

[9] J. P. May, J. McClure and G. Triantafillou, Equivariant localization, Bull. London Math. Soc., 14 (1982), 223-230.

[10] T. Matumoto, On $G-C W$ complexes and a theorem of J. H. C. Whitehead. J. Fac. Sci. Univ. Tokyo Sect. IA Math., 18 (1971/72), 363-374.

[11] J. F. McClendon, Obstruction theory in fiber spaces, Math. Z., 120 (1971), 1-17.

[12] C. Morgan and R. Piccinini, Fibrations, Expositiones Math., 4 (1986), 217-242.

[13] J. M. Møller, Spaces of sections of Eilenberg-Mac Lane fibrations, Pacific J. Math., 130 (1987), 171-186.

[14] _ Nilpotent spaces of sections, Trans. Amer. Math. Soc., 303 (1987), 733741.

[15] R. Schultz, Homotopy decompositions of equivariant function spaces, Math. Z., 131 (1973), 49-75 and Math. Z., 132 (1973), 69-90.

[16] R. Thom, L'homologie des espaces fonctionelles, Colloque de topologie algebrique, Louvain 1956. Thone, Liege; Masson, Paris, 1957, 29-39.

[17] E. Thomas, Seminar on Fiber Spaces. Lecture Notes in Mathematics 13. Springer-Verlag, Berlin, Heidelberg, New York 1966.

[18] G. V. Triantafillou, Equivariant minimal models, Trans. Amer. Math. Soc., 274 (1982), 509-532.

[19] S. Waner, Equivariant homotopy theory and Milnor's theorem, Trans. Amer. Math. Soc., 258 (1980), 351-369.

[20] G. W. Whitehead, Elements of Homotopy Theory, Graduate Texts in Mathematics 61. Springer-Verlag, New York, Heidelberg, Berlin 1978.

[21] H. Scheerer, Localisation des espaces de sections et de retractions, C. R. Acad. Sc. Paris, 287 (1978), 851-853.

Received February 4, 1988 and in revised form, April 3, 1989.

Mathematical INSTitute

UNIVERSITETSPARKEN 5

DK-2100 København $\varnothing$, Denmark 



\section{PACIFIC JOURNAL OF MATHEMATICS EDITORS}

\author{
V. S. VARADARAJAN \\ (Managing Editor) \\ University of California \\ Los Angeles, CA 90024-1555-05 \\ Herbert Clemens \\ University of Utah \\ Salt Lake City, UT 84112 \\ ThOMAS ENRIGHT \\ University of California, San Diego \\ La Jolla, CA 92093
}

\section{R. FINN}

Stanford University

Stanford, CA 94305

HeRmann FlaschKa

University of Arizona

Tucson, AZ 85721

VAUGHAN F. R. JONES

University of California

Berkeley, CA 94720

Steven KerckhofF

Stanford University

Stanford, CA 94305

\author{
RobION KIRBY \\ University of California \\ Berkeley, CA 94720 \\ C. C. Moore \\ University of California \\ Berkeley, CA 94720 \\ HAROLD STARK \\ University of California, San Diego \\ La Jolla, CA 92093
}

\section{ASSOCIATE EDITORS}
R. ARENS
E. F. BECKenBach
B. H. NeumanN
F. WOLF
K. YoshidA (1906-1982)
(1904-1989)

\section{SUPPORTING INSTITUTIONS}

UNIVERSITY OF ARIZONA

UNIVERSITY OF BRITISH COLUMBIA

CALIFORNIA INSTITUTE OF TECHNOLOGY

UNIVERSITY OF CALIFORNIA

MONTANA STATE UNIVERSITY

UNIVERSITY OF NEVADA, RENO

NEW MEXICO STATE UNIVERSITY

OREGON STATE UNIVERSITY
UNIVERSITY OF OREGON

UNIVERSITY OF SOUTHERN CALIFORNIA

STANFORD UNIVERSITY

UNIVERSITY OF HAWAII

UNIVERSITY OF TOKYO

UNIVERSITY OF UTAH

WASHINGTON STATE UNIVERSITY

UNIVERSITY OF WASHINGTON 


\section{Pacific Journal of Mathematics}

Vol. 142, No. $1 \quad$ January, 1990

Marco Andreatta, Mauro Beltrametti and Andrew Sommese, Generic properties of the adjuction mapping for singular surfaces and applications

Chen-Lian Chuang and Pjek-Hwee Lee, On regular subdirect products of

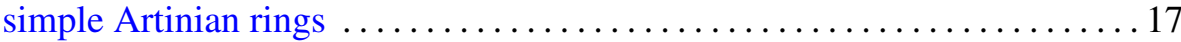

Fernando Giménez and Vicente Miquel Molina, Volume estimates for real hypersurfaces of a Kaehler manifold with strictly positive holomorphic sectional and antiholomorphic Ricci curvatures $\ldots \ldots \ldots \ldots \ldots \ldots 23$

Richard J. Griego and Andrzej Korzeniowski, Asymptotics for certain Wiener integrals associated with higher order differential operators

Abdeslam Mesnaoui, Unitary bordism of classifying spaces of quaternion

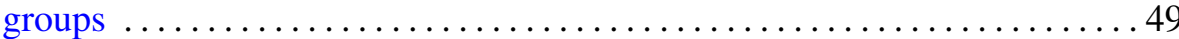

Abdeslam Mesnaoui, Unitary cobordism of classifying spaces of quaternion

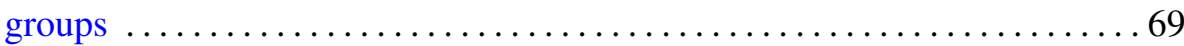

Jesper M. Møller, On equivariant function spaces $\ldots \ldots \ldots \ldots \ldots \ldots \ldots \ldots$

Bassam Nassrallah, A $q$-analogue of Appell's $F_{1}$ function, its integral representation and transformations

Peter A Ohring, Solvability of invariant differential operators on metabelian

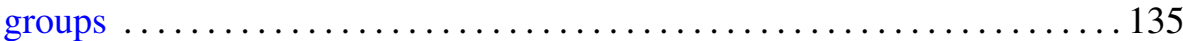

Athanase Papadopoulos and R. C. Penner, Enumerating pseudo-Anosov foliations

Ti-Jun Xiao and Liang Jin, On complete second order linear differential

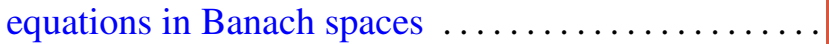

Carl Widland and Robert F. Lax, Weierstrass points on Gorenstein curves 